\title{
El constructo Enfoques de Aprendizaje: un análisis bibliométrico de las publicaciones en español en los últimos 20 años*
}

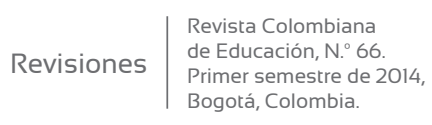

\section{//The Construct Learning Approaches: A Bibliometric Analysis Of The Publications In Spanish In The Last 20 Years}

\section{//O constructo Enfoques de Aprendizagem: uma análise bibliométrico das publicações em espanhol nos últimos 20 anos}

\author{
Manuel Guillermo Soler Contreras **
}

Recibido: 27/12/2013 Evaluado: $23 / 02 / 2014$ $17 / 03 / 2014$

\begin{abstract}
Esta revisión bibliométrica se ha realizado en el marco de la investigación que sobre enfoques de aprendizaje y de enseñanza adelanta el autor, en el énfasis de Ciencias, Acciones y Creencias del Doctorado Interinstitucional en Educación, en la Universidad Pedagógica Nacional.

Doctorando del Doctorado Interinstitucional en Educación UPN. Magíster en Didáctica de las Ciencias. Especialista en Análisis Químico Instrumental. Licenciado en Química. Docente Secretaría de Educación de Soacha, Colombia. Correo electrónico: maguiso5@hotmail.com
\end{abstract}

\section{Resumen}

En este artículo se presenta una revisión bibliométrica de las principales publicaciones que centran su atención en el constructo enfoques de aprendizaje en las principales revistas científicas en español, de España y Latinoamérica. Se trata de un análisis retrospectivo que cubre el periodo comprendido entre 1993 y 2013, durante el cual se tuvo en cuenta el número total de artículos que han tratado el tema, las revistas en las cuales han sido publicados y los autores con mayor número de investigaciones. Así mismo, se establecen categorías relacionadas con los temas específicos objeto de investigación y se plantean algunas relaciones entre los enfoques de aprendizaje y el rendimiento académico de estudiantes universitarios. El análisis de los resultados conduce, entre otras, a las siguientes conclusiones: durante los últimos veinte años hubo un crecimiento paulatino de artículos, los cuales alcanzaron una alta cifra entre 2009 y 2011. Se destacan además, como autores de mayor productividad académica en el tema los doctores Fuensanta Hernández Pina y Alfonso Barca Lozano, y como temas de investigación la caracterización de los enfoques de aprendizaje y su diferenciación con otros constructos semejantes. El análisis estadístico que se muestra en algunos artículos, dejan ver que el enfoque profundo se relaciona positivamente con el rendimiento académico de estudiantes universitarios.

\section{Abstract}

This article shows a bibliometric review of the main publications that are focused in the construct learning approaches in the most important scientific magazines written in Spanish at the Iberian Peninsula and Latin America; It is about a retrospective analysis that covers the period between 1993 and 2013. In this lapse of time it shows the total amount of articles that have faced with the topic, the magazines that have been published and it emphasizes the authors with a major number of publications. At the same time it establishes categories related to specific topics of investigation, besides it establishes some relations between the learning approaches and the academic performance of university students. The analysis of the results leads to, among others, to these conclusions: during the study period there is a slightly grown in the number of articles published reaching a maximum between 2009 and 2011. Besides the author outlined

\section{Palabras Clave}

Enfoques de aprendizaje, rendimiento académico, enseñanza, motivación, estrategia.

\section{Keywords}

Learning approaches, academic development, teaching, motivation, strategy.

\section{Palavras chave}

Enfoques de aprendizagem, desempenho académico, ensino, motivação, estratégia. 
with the maximum of academic productivity in the topic are the doctors Fuensanta Hernández and Alfonso Barca; and the objective topics characterizes the learning approaches and its differentiation with the similar constructs. The statistics studies show that the deep approach correlates positively with the academic performance of university students.

\section{Resumo}

O artigo apresenta uma revisão bibliométrica das principais publicações sobre o construto enfoques de aprendizagem nas principais revistas científicas em espanhol, da Espanha e a América Latina. Foi feita uma análise retrospectiva abrangendo o período entre 1993 e 2013, tendo em conta o número total de artigos que têm trabalhado nesse assunto, as revistas onde foram publicados e os autores com maior número de pesquisas nessa área. Igualmente, estabeleceu-se um conjunto de categorias relacionadas com os temas de pesquisa e definiram-se algumas relações entre os enfoques de aprendizagem e o desempenho académico de estudantes universitários. Algumas conclusões obtidas são: Durante os últimos vinte anos teve um aumento gradual no número de artigos produzidos, especialmente durante os anos 2009 e 2011. Destacam-se como os autores com maior produção na área os doutores Fuensanta Hernández Pina e Alfonso Barca Lozano, e como principais temas de pesquisa a caracterização dos enfoques de aprendizagem e sua diferenciação de outros construtos semelhantes. A análise estatística apresentada em alguns artigos sugere que o enfoque profundo tem relação positiva com o desempenho académico de estudantes universitários.

En la línea de investigación Student Approache to Learning (SAL) emerge como uno de sus pilares teóricos el constructo enfoques de aprendizaje (Marton y Säljö, 1976a, 1976b), el cual hace referencia a la manera como los estudiantes orientan sus procesos de estudio, ya sea mediante un enfoque de aprendizaje superficial o profundo. Uno u otro no constituye una propiedad estable de los estudiantes, pues depende en gran medida de los factores contextuales y de la naturaleza de la tarea académica que ellos enfrentan (Biggs, 2005). Por esta razón, las variables que determinan el enfoque de aprendizaje son: la motivación (intrínseca para el enfoque profundo o extrínseca para el superficial) y las estrategias (de alto nivel cognitivo en el enfoque profundo o de bajo nivel cognitivo en el superficial).

El análisis bibliométrico que acá se presenta, recoge las evidencias de las investigaciones que sobre los enfoques de aprendizaje han surgido en español, y de su contribución al rendimiento académico de los estudiantes.

Se parte de la construcción de una base de datos, para lo cual se ha recurrido, como fuentes de información, a Scielo, dialnet, redalyc y a las URL de las diversas revistas que sobre educación centran sus publicaciones de Latinoamérica, el Caribe y España; además, se ha consultado el reporte de índice de impacto de 
In-Recs. Para este análisis se ha tomado como referencia un periodo de veinte años (1993 y 2013).

\section{Revistas destacadas en publicaciones sobre enfoques de aprendizaje}

Los artículos en idioma español, que sobre el constructo enfoques de aprendizaje se han detectado a partir de 1993, han sido en total 54. De estos se aglutinan en mayor porcentaje en España con 41 artículos $(76 \%)$, seguido muy de lejos por
Colombia con $6(11 \%)$ y Chile con $3(5 \%)$; hay otros países en los que solo se ha encontrado un artículo (figura 1).

La gran cantidad de publicaciones en España se puede explicar por el número de revistas relacionadas con la psicología y la educación, y porque desde la última década del siglo XX, empezaron a surgir investigadores que se interesaron por centrar sus trabajos en la línea SAL en el contexto español. En la tabla 1 se relacionan las revistas españolas que han publicado artículos sobre los enfoques de aprendizaje.

Figura 1. Artículos sobre enfoques de aprendizaje en idioma español.
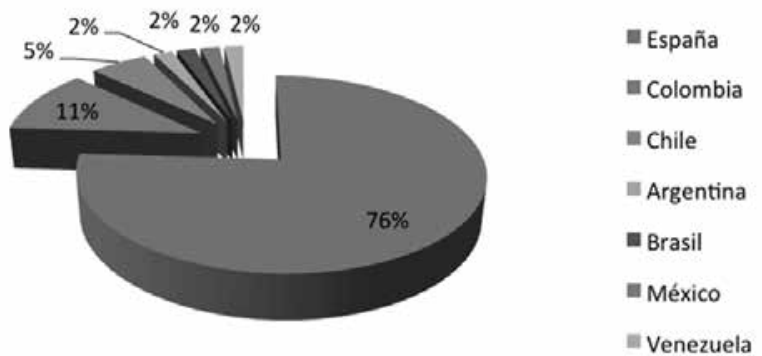

Tabla 1. Revistas españolas que han publicado artículos sobre enfoques de aprendizaje.

\begin{tabular}{|c|c|}
\hline ÍTEM & REVISTA \\
\hline 1 & Revista de Investigación Educativa \\
\hline 2 & Revista Galego-portuguesa de Psicoloxía e educación \\
\hline 3 & Psicoterma \\
\hline 4 & Revista de orientación educativa vocacional \\
\hline 5 & Revista interuniversitaria de formación del profesorado \\
\hline 6 & Pixel-Bit Revista de medios y Educación \\
\hline 7 & Revista Iberoamericana de Educación \\
\hline 8 & Revista de Estudios e Experiencia Educativas \\
\hline 9 & \\
\hline
\end{tabular}




\begin{tabular}{|c|c|}
\hline ÍTEM & REVISTA \\
\hline 10 & Revista de Investigación en Educación \\
\hline 11 & Revista de Psicodidáctica \\
\hline 12 & Aula abierta \\
\hline 13 & Revista Española de Pedagogía \\
\hline 14 & Revista Estilos de Aprendizaje \\
\hline 15 & Revista Española de orientación y Psicopedagogía \\
\hline
\end{tabular}

Las revistas de países latinoamericanos que han registrado artículos sobre el tema que se convoca aquí, se relacionan en la tabla 2. Se resalta la cantidad de revistas especializadas que en Colombia se han publicado sobre enfoques de aprendizaje (seis revistas diferentes) comparado con otros países de la región como Chile (dos revistas diferentes) y Brasil, Argentina, Venezuela y México (una revista cada uno).

Tabla 2. Revistas latinoamericanas que han publicado artículos sobre enfoques de aprendizaje.

\begin{tabular}{|c|c|c|}
\hline ÍTEM & REVISTA & PAís \\
\hline 1 & Acta Médica Colombiana & Colombia \\
\hline 2 & Folios & Colombia \\
\hline 3 & Biosalud & Colombia \\
\hline 4 & Revista Colombiana de Educación & Colombia \\
\hline 5 & Universitas Psichologica & Colombia \\
\hline 6 & Revista Científica & Colombia \\
\hline 7 & Calidad en la Educación & Chile \\
\hline 8 & Estudios pedagógicos & Chile \\
\hline 9 & Revista AMAzônica & Brasil \\
\hline 10 & Cuadernos de Educación & Argentina \\
\hline 11 & Revista de Pedagogía & Venezuela \\
\hline 12 & Medigraphic & México \\
\hline
\end{tabular}

De todas las revistas consultadas, la que publica una mayor cantidad de artículos (16) relacionados con la temática de interés en este documento, es la Revista de Investigación Educativa (RIE) de España (tabla 3).

Tabla 3. Artículos sobre enfoques de aprendizaje publicados en la Revista de Investigación Educativa.

\begin{tabular}{|c|c|c|c|c|c|}
\hline ÍtEM & AÑO & VOL. & No & \multicolumn{1}{|c|}{ TítULO DEL ARTíCULO } \\
\hline 1 & 2001 & 19 & 2 & $\begin{array}{l}\text { Los enfoques de aprendizaje en estudiantes universitarios espa- } \\
\text { ñoles }\end{array}$ \\
\hline 2 & 2001 & 20 & 2 & $\begin{array}{l}\text { La investigación sobre diagnóstico de los estilos de aprendizaje } \\
\text { en la enseñanza superior }\end{array}$ \\
\hline
\end{tabular}




\begin{tabular}{|c|c|c|c|c|}
\hline ÍTEM & AÑO & VOL. & No & TÍTULO DEL ARTíCULO \\
\hline 3 & 2002 & 20 & 2 & $\begin{array}{l}\text { Consistencia entre motivos y estrategias de aprendizaje en estu- } \\
\text { diantes universitarios }\end{array}$ \\
\hline 4 & 2002 & 20 & 2 & $\begin{array}{l}\text { El género: ¿constructo mediador en los enfoques de aprendizaje } \\
\text { universitario? }\end{array}$ \\
\hline 5 & 2003 & 21 & 2 & $\begin{array}{l}\text { Estudio transcultural de los enfoques de aprendizaje en Educa- } \\
\text { ción Superior }\end{array}$ \\
\hline 6 & 2005 & 23 & 2 & $\begin{array}{l}\text { Enfoques de aprendizaje y rendimiento académico de los estu- } \\
\text { diantes universitarios }\end{array}$ \\
\hline 7 & 2006 & 24 & 2 & $\begin{array}{l}\text { Promoción del aprendizaje estratégico y competencias de apren- } \\
\text { dizaje en estudiantes de primero de universidad: Evaluación de } \\
\text { una intervención }\end{array}$ \\
\hline 8 & 2006 & 24 & 2 & $\begin{array}{l}\text { Vinculación de los enfoques de aprendizaje con los intereses pro- } \\
\text { fesionales y los rasgos de personalidad. Aportaciones a la inno- } \\
\text { vación del proceso de enseñanza y aprendizaje en la educación } \\
\text { superior }\end{array}$ \\
\hline 9 & 2007 & 25 & 2 & $\begin{array}{l}\text { Estrategias de aprendizaje y rendimiento académico en estudian- } \\
\text { tes universitarios }\end{array}$ \\
\hline 10 & 2008 & 26 & 2 & $\begin{array}{l}\text { Enfoques de aprendizaje y rendimiento institucional y afectivo de } \\
\text { los alumnos de la titulación de ciencias de la actividad física y } \\
\text { del deporte }\end{array}$ \\
\hline 11 & 2009 & 27 & 2 & $\begin{array}{l}\text { Rendimiento académico y enfoques de aprendizaje: una apro- } \\
\text { ximación a la realidad de la enseñanza superior brasileña en la } \\
\text { Región Norte }\end{array}$ \\
\hline 12 & 2011 & 29 & 2 & $\begin{array}{l}\text { Estructura latente y Consistencia interna del R-SPQ-2F: Reinter- } \\
\text { pretando los enfoques de aprendizaje en el EEES }\end{array}$ \\
\hline 13 & 2012 & 30 & 1 & $\begin{array}{l}\text { El ocio y los enfoques de aprendizaje en estudiantes universita- } \\
\text { rios }\end{array}$ \\
\hline 14 & 2013 & 31 & 2 & $\begin{array}{l}\text { Enfoques de aprendizaje en estudiantes universitarios: compara- } \\
\text { ción de resultados con los cuestionarios ASSIST y R-SPQ- } 2 \text { F }\end{array}$ \\
\hline 15 & 2013 & 31 & 2 & $\begin{array}{l}\text { Enfoques de aprendizaje de los estudiantes y metodología } \\
\text { docente: Evolución hacia el nuevo sistema de formación e inte- } \\
\text { racción propuesta en el EEES. }\end{array}$ \\
\hline 16 & 2013 & 31 & 2 & $\begin{array}{l}\text { Valoración de las TIC por los estudiantes universitarios y su rela- } \\
\text { ción con los enfoques de aprendizaje }\end{array}$ \\
\hline
\end{tabular}

En la tabla 4 se relacionan, en segundo lugar de publicaciones, los seis artículos de la Revista Galego-portuguesa de Psicoloxía e educación; y en la tabla 5, los cinco artículos publicados en Psicoterma, tercera revista en número de publicaciones. Las tres revistas relacionadas anteriormente son de España. 
Tabla 4. Artículos sobre enfoques de aprendizaje publicados en la Revista Galego-portuguesa de Psicoloxía e educación.

\begin{tabular}{|c|c|c|c|c|}
\hline ÍTEM & AÑO & VOL & No & TíTULO DEL ARTíCULO \\
\hline 1 & 2000 & 6 & 4 & $\begin{array}{l}\text { Enfoques de aprendizaje, estilos atribucionales y rendimiento } \\
\text { académico en una muestra de alumnos de educación secunda- } \\
\text { ria de Brasil }\end{array}$ \\
\hline 2 & 2000 & 6 & 4 & $\begin{array}{l}\text { Factores de atribución causal, enfoques de aprendizaje y rendi- } \\
\text { miento académico en el alumnado de educación secundaria de } \\
\text { Galicia: Datos para un análisis correlacional }\end{array}$ \\
\hline 3 & 2003 & 8 & 7 & Familia, centro y enfoques de aprendizaje \\
\hline 4 & 2006 & 13 & 12 & $\begin{array}{l}\text { Un modelo bifactorial para la explicación de los motivos y estra- } \\
\text { tegias de aprendizaje en las tareas de estudio con alumnos de } \\
\text { educación secundaria. }\end{array}$ \\
\hline 5 & 2010 & 18 & 1 & $\begin{array}{l}\text { Enfoques de aprendizaje y rendimiento académico en educación } \\
\text { secundaria }\end{array}$ \\
\hline 6 & 2011 & 19 & 2 & $\begin{array}{l}\text { Autoconcepto y enfoques de aprendizaje: sus efectos en el ren- } \\
\text { dimiento académico en alumnado universitario de Republica } \\
\text { dominicana }\end{array}$ \\
\hline
\end{tabular}

Tabla 5. Artículos sobre enfoques de aprendizaje publicados en Psicoterma.

\begin{tabular}{|c|c|c|c|c|}
\hline ÍTEM & AÑO & VOL & No & TíTULO DEL ARTíCULO \\
\hline 1 & 1998 & 10 & 2 & $\begin{array}{l}\text { Variables cognitivo-motivacionales, enfoques de aprendizaje y } \\
\text { rendimiento académico }\end{array}$ \\
\hline 2 & 2000 & 12 & 3 & Enfoques de aprendizaje en estudiantes universitarios \\
\hline 3 & 2000 & 10 & 3 & Diferencias de género en estrategias y estilos de aprendizaje \\
\hline 4 & 2004 & 16 & 1 & $\begin{array}{l}\text { Atribuciones causales y enfoques de aprendizaje: la escala } \\
\text { SIACEPA }\end{array}$ \\
\hline 5 & 2008 & 20 & 4 & $\begin{array}{l}\text { Enfoques de aprendizaje, autorregulación y rendimiento } \\
\text { en tres universidades europeas }\end{array}$ \\
\hline
\end{tabular}




\section{Autores destacados en publicaciones sobre enfoques de aprendizaje}

Tras el consolidado de la información de las tablas precedentes, se ha encontrado que Fuensanta Hernández Pina, directora del Departamento de Métodos de Investigación y Diagnóstico Educativo de la Facultad de Educación de la Universidad de Murcia, es quien lidera con nueve publicaciones como autora o coautora (tabla 6). Le sigue Alfonso Barca Lozano del Departamento de Psicología Evolutiva y de la Educación, de la Facultad de Educación, de la Universidad de la Coruña, con siete publicaciones como autor o coautor. En tercer lugar se encuentra el autor Javier Maquilón Sánchez del Departamento de Métodos de Investigación y Diagnóstico Educativo de la Facultad de Educación de la Universidad de Murcia, con cuatro publicaciones.

Tabla 6. Autores que lideran en número de publicaciones sobre enfoques de aprendizaje.

\begin{tabular}{|c|c|}
\hline AUTOR & ARTíCULOS \\
\hline Fuensanta Hernández Pina & 9 \\
\hline Alfonso Barca Lozano & 7 \\
\hline Javier J. Maquilón Sánchez & 4 \\
\hline Eva María Olmedo Moreno & 3 \\
\hline
\end{tabular}

Al indagar en detalle la tradición de investigación de los primeros dos autores, se hace importante resaltar la labor académica de Fuensanta Hernández Pina, no solo por liderar en número de publicaciones sobre enfoques de aprendizaje, sino por ser la pionera en esta línea de investigación en idioma español, pues fue ella quien en 1993 inició esta tradición y quien, de manera constante durante estos últimos veinte años, no ha suspendido la investigación acerca este tema. Ya Ariza y Quevedo (2013) reportaban a esta autora como la más citada en In-Recs (1996-2009) con un total de 34 citas.

De otro lado, Hernández Pina realiza también una ardua investigación en otra línea complementaria como es la de los enfoques de enseñanza de los profesores (Hernández, Maquilón y Monroy, 2012); sus investigaciones son de gran interés dado que no se ha limitado a una franja específica de la educación, por el contrario, ha generado investigación en los diversos ámbitos educativos desde la primaria hasta la universitaria y en diversos contextos de Murcia, España y algunos países latinoamericanos. Es ella, en gran medida, responsable de la formación de muchos de los investigadores de América Latina, como directora de sus tesis doctorales; ellos han contribuido a diseminar esta tradición investigadora en nuestro continente. La tabla 7 reporta los artículos que como autora o coautora ha publicado esta investigadora. 
Tabla 7. Artículos sobre enfoques de aprendizaje publicados con la autoría o coautoría de Fuensanta Hernández Pina.

\begin{tabular}{|c|c|c|c|c|c|c|c|}
\hline \multirow{7}{*}{ 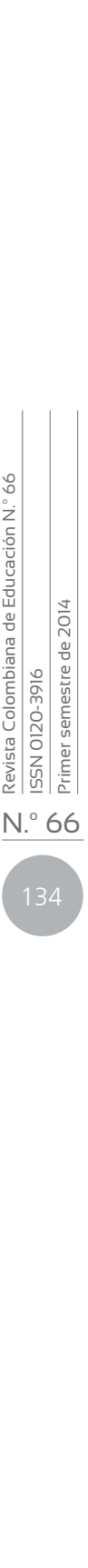 } & ÍTEM & REVISTA & AÑO & VOL & No & TÍTULO DEL ARTÍCULO & AUTORES \\
\hline & 1 & $\begin{array}{l}\text { Revista de Investi- } \\
\text { gación Educativa }\end{array}$ & 2001 & 19 & 2 & $\begin{array}{l}\text { Los enfoques de aprendi- } \\
\text { zaje en estudiantes uni- } \\
\text { versitarios Españoles }\end{array}$ & $\begin{array}{l}\text { Abalde Paz, Miguel } \\
\text { Muñoz Cantero, } \\
\text { Colbs, Leonor } \\
\text { Buendía Eisman, } \\
\text { Eva María Olmedo } \\
\text { Moreno y Emilio } \\
\text { Berrocal de Luna } \\
\text { Fuensanta Hernán- } \\
\text { dez Pina, Javier J. } \\
\text { Maquilón Sánchez } \\
\text { y otros colabor- } \\
\text { dores. }\end{array}$ \\
\hline & 2 & $\begin{array}{l}\text { Revista de Investi- } \\
\text { gación Educativa }\end{array}$ & 2002 & 20 & 2 & $\begin{array}{l}\text { Consistencia entre moti- } \\
\text { vos y estrategias de apren- } \\
\text { dizaje en estudiantes uni- } \\
\text { versitarios }\end{array}$ & $\begin{array}{l}\text { Fuensanta Hernán- } \\
\text { dez Pina, María Paz } \\
\text { García Sanz, Pilar } \\
\text { Martínez Clares, } \\
\text { Rosa María Her- } \\
\text { vás Avilés, Javier } \\
\text { Maquilón Sánchez }\end{array}$ \\
\hline & 3 & $\begin{array}{l}\text { Revista de Investi- } \\
\text { gación Educativa }\end{array}$ & 2006 & 24 & 2 & $\begin{array}{l}\text { Promoción del aprendi- } \\
\text { zaje estratégico y compe- } \\
\text { tencias de aprendizaje en } \\
\text { estudiantes de primero de } \\
\text { universidad: Evaluación } \\
\text { de una intervención }\end{array}$ & $\begin{array}{l}\text { Fuensanta Hernán- } \\
\text { dez Pina, } \\
\text { Pedro Rosario, } \\
\text { José David Cuesta } \\
\text { de Tejada, } \\
\text { Pilar Martínez } \\
\text { Clares, } \\
\text { Encarna Ruiz Lara }\end{array}$ \\
\hline & 4 & $\begin{array}{l}\text { Revista de Investi- } \\
\text { gación Educativa }\end{array}$ & 2008 & 26 & 2 & $\begin{array}{l}\text { Enfoques de aprendizaje } \\
\text { y rendimiento institucio- } \\
\text { nal y afectivo de los alum- } \\
\text { nos de la titulación de } \\
\text { ciencias de la actividad } \\
\text { física y del deporte }\end{array}$ & $\begin{array}{l}\text { Encarnación Ruiz } \\
\text { Lara, Fuensanta } \\
\text { Hernández Pina, } \\
\text { Fernando Ureña } \\
\text { Villanueva }\end{array}$ \\
\hline & 5 & $\begin{array}{l}\text { Revista de Investi- } \\
\text { gación Educativa }\end{array}$ & 2013 & 31 & 2 & $\begin{array}{l}\text { Valoración de las TIC por } \\
\text { los estudiantes universita- } \\
\text { rios y su relación con los } \\
\text { enfoques de aprendizaje }\end{array}$ & $\begin{array}{l}\text { Javier J. Maqui- } \\
\text { lón Sánchez, Ana } \\
\text { Belén Mirete Ruiz, } \\
\text { Francisco Alberto } \\
\text { García Sánchez, } \\
\text { Fuensanta Hernán- } \\
\text { dez Pina }\end{array}$ \\
\hline & 6 & $\begin{array}{l}\text { Revista de orien- } \\
\text { tación educativa } \\
\text { vocacional }\end{array}$ & 1993 & 4 & 5 & $\begin{array}{l}\text { Evaluación de los enfo- } \\
\text { ques de aprendizaje en } \\
\text { alumnos de octavo de } \\
\text { E.G.B. }\end{array}$ & $\begin{array}{l}\text { Fuensanta Hernán- } \\
\text { dez Pina }\end{array}$ \\
\hline
\end{tabular}




\begin{tabular}{|c|c|c|c|c|c|c|}
\hline ÍTEM & REVISTA & AÑO & VOL & No & TÍTULO DEL ARTÍCULO & AUTORES \\
\hline 7 & Revista Fuentes & 2004 & 6 & & $\begin{array}{l}\text { Análisis del cuestiona- } \\
\text { rio de proceso de estu- } \\
\text { dio } 2 \text { factores de Biggs en } \\
\text { estudiantes universitarios } \\
\text { españoles }\end{array}$ & $\begin{array}{l}\text { Fuensanta Hernán- } \\
\text { dez Pina, } \\
\text { María Paz García } \\
\text { Sanz, } \\
\text { Javier Maquilón } \\
\text { Sánchez }\end{array}$ \\
\hline 8 & $\begin{array}{l}\text { Revista Iberoameri- } \\
\text { cana de Educación }\end{array}$ & 2010 & 53 & 7 & $\begin{array}{l}\text { Enfoques de aprendizaje } \\
\text { en estudiantes universi- } \\
\text { tarios }\end{array}$ & $\begin{array}{l}\text { Fuensanta Hernán- } \\
\text { dez Pina, María } \\
\text { Concepción Rodrí- } \\
\text { guez Nieto, Encar- } \\
\text { nación Ruiz Lara, } \\
\text { Jesús Enrique Esqui- } \\
\text { vel Cruz }\end{array}$ \\
\hline 9 & $\begin{array}{l}\text { Revista Española } \\
\text { de orientación y } \\
\text { Psicopedagogía }\end{array}$ & 2001 & 12 & 2 & $\begin{array}{l}\text { Estudio empírico de los } \\
\text { enfoques de aprendizaje } \\
\text { de los estudiantes uni- } \\
\text { versitarios en función } \\
\text { del perfil de su titulación } \\
\text { (profundo vs superficial) }\end{array}$ & $\begin{array}{l}\text { Fuensanta Hernán- } \\
\text { dez Pina, García M. } \\
\text { P. Maquilon Sán- } \\
\text { chez, J. J. }\end{array}$ \\
\hline
\end{tabular}

Por su parte, Alfonso Barca Lozano se destaca también por su contribución en la investigación sobre enfoques de aprendizaje en idioma español, pues desde comienzos del siglo XXI, ha venido generando una prolífica investigación, que de igual modo, ha trascendido las fronteras españolas difundiéndose ante todo hacia Brasil (tabla 8).

Tabla 8. Artículos sobre enfoques de aprendizaje publicados con la autoría o coautoría Alfonso Barca Lozano.

\begin{tabular}{|c|c|c|c|c|c|c|}
\hline ÍTEM & REVISTA & AÑO & VOL. & No & TÍTULO DEL ARTÍCULO & AUTORES \\
\hline 1 & $\begin{array}{l}\text { Revista de Investi- } \\
\text { gación Educativa }\end{array}$ & 2009 & 27 & 2 & $\begin{array}{l}\text { Rendimiento académico y } \\
\text { enfoques de aprendizaje: } \\
\text { una aproximación a la } \\
\text { realidad de la enseñanza } \\
\text { superior brasileña en la } \\
\text { Región Norte }\end{array}$ & $\begin{array}{l}\text { Eduardo Abalde } \\
\text { Paz, Alfonso Barca } \\
\text { Lozano, Jesús } \\
\text { Miguel Muñoz } \\
\text { Cantero, Marcos } \\
\text { Fernando Ziemer }\end{array}$ \\
\hline 2 & $\begin{array}{l}\text { Revista Galego- } \\
\text { portuguesa de } \\
\text { Psicoloxía e edu- } \\
\text { cación }\end{array}$ & 2000 & 6 & 4 & $\begin{array}{l}\text { Enfoques de aprendizaje, } \\
\text { estilos atribucionales y } \\
\text { rendimiento académico } \\
\text { en una muestra de alum- } \\
\text { nos de educación secun- } \\
\text { daria de Brasil }\end{array}$ & $\begin{array}{l}\text { Alfonso Barca } \\
\text { Lozano, } \\
\text { Celia Regina } \\
\text { Pesutti, } \\
\text { Juan Carlos Brenlla } \\
\text { Blanco, } \\
\text { Silvia Santamaría } \\
\text { Canosa }\end{array}$ \\
\hline
\end{tabular}




\begin{tabular}{|c|c|c|c|c|c|c|}
\hline ÍTEM & REVISTA & AÑO & VOL. & No & TÍTULO DEL ARTÍCULO & AUTORES \\
\hline 3 & $\begin{array}{l}\text { Revista Galego- } \\
\text { portuguesa de } \\
\text { Psicoloxía e edu- } \\
\text { cación }\end{array}$ & 2000 & 6 & 4 & $\begin{array}{l}\text { Factores de atribu- } \\
\text { ción causal, enfoques } \\
\text { de aprendizaje y rendi- } \\
\text { miento académico en } \\
\text { el alumnado de educa- } \\
\text { ción secundaria de Gali- } \\
\text { cia: Datos para un análisis } \\
\text { correlaconal }\end{array}$ & $\begin{array}{l}\text { Alfonso Barca } \\
\text { Lozano, } \\
\text { Celia Regina } \\
\text { Pesutti, } \\
\text { Juan Carlos Brenlla } \\
\text { Blancol, } \\
\text { Silvia Santamaría } \\
\text { Canosa }\end{array}$ \\
\hline 4 & $\begin{array}{l}\text { Revista Galego- } \\
\text { portuguesa de } \\
\text { Psicoloxía e edu- } \\
\text { cación }\end{array}$ & 2006 & 13 & 12 & $\begin{array}{l}\text { Un modelo bifactorial } \\
\text { para la explicación de los } \\
\text { motivos y estrategias de } \\
\text { aprendizaje en las tareas } \\
\text { de estudio con alumnos } \\
\text { de educación secundaria. }\end{array}$ & $\begin{array}{l}\text { Alfonso Barca } \\
\text { Lozano, } \\
\text { Juan C. Brenlla } \\
\text { Blanco }\end{array}$ \\
\hline 5 & $\begin{array}{l}\text { Revista Galego- } \\
\text { portuguesa de } \\
\text { Psicoloxía e edu- } \\
\text { cación }\end{array}$ & 2011 & 19 & 2 & $\begin{array}{l}\text { Autoconcepto y enfoques } \\
\text { de aprendizaje: sus efec- } \\
\text { tos en el rendimiento aca- } \\
\text { démico en alumnado uni- } \\
\text { versitario de Republica } \\
\text { Dominicana }\end{array}$ & $\begin{array}{l}\text { Alfonso Barca } \\
\text { Lozano, } \\
\text { Amparo Fernández } \\
\text { de Mejía, } \\
\text { Radhamés Mejía }\end{array}$ \\
\hline 6 & Psicoterma & 2004 & 16 & 1 & $\begin{array}{l}\text { Atribuciones causales y } \\
\text { enfoques de aprendizaje: } \\
\text { la escala SIACEPA }\end{array}$ & $\begin{array}{l}\text { Alfonso Barca } \\
\text { Lozano, Manuel } \\
\text { Peralbo Uzquiano y } \\
\text { Juan Carlos Brenlla } \\
\text { Blanco }\end{array}$ \\
\hline 7 & Revista AMAzônica & 2008 & 1 & 1 & $\begin{array}{l}\text { Motivación y aprendizaje } \\
\text { en el alumnado de edu- } \\
\text { cación secundaria y ren- } \\
\text { dimiento académico: un } \\
\text { análisis desde la diversi- } \\
\text { dad e inclusión educativa }\end{array}$ & $\begin{array}{l}\text { Alfonso Barca } \\
\text { Lozano, } \\
\text { Suely a. Do Nasci- } \\
\text { mento } \\
\text { Juan Carlos Bren- } \\
\text { Ila, Ana María Porto } \\
\text { Rioboo y Eduardo } \\
\text { Barca Enríquez }\end{array}$ \\
\hline
\end{tabular}

Analizando el periodo comprendido entre 1993 y 2013 (figura 2), se observa un aumento progresivo en el número de publicaciones, y que en 2009 y 2011 es cuando han surgido mayor número de artículos, seis en cada año. Por su parte, a finales del siglo pasado se encontraron muy pocos documentos en idioma español sobre los enfoques de aprendizaje. 
Figura 2. Publicación de artículos sobre enfoques de aprendizaje por año (1993 - 2013).

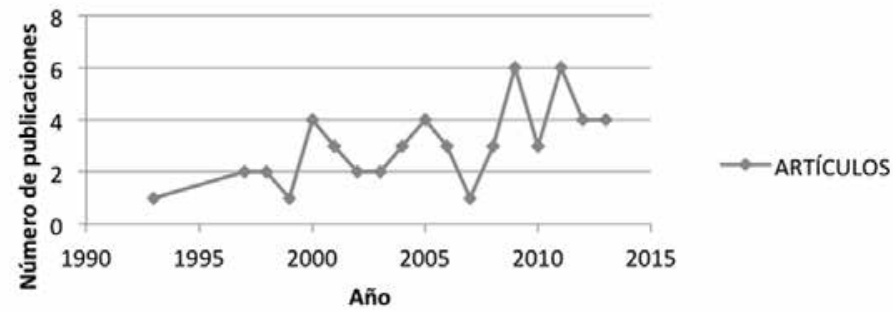

\section{Categorías de análisis en los artículos sobre enfoques de aprendizaje}

Luego de realizar un barrido por todas las revistas y autores, que en los últimos veinte años han tenido como objeto de interés los enfoques de aprendizaje en idioma español, se analiza la naturaleza de estas investigaciones y su repercusión en la calidad educativa. Para ello, se han tomado los 54 artículos, y luego de una minuciosa revisión, se han definido un total de 12 categorías sobre las que se enfocan estas investigaciones. Estas categorías se relacionan en la tabla 9, junto con el número de artículos que orientan su interés en relacionar la categoría correspondiente con el constructo enfoques de aprendizaje.

Tabla 9. Categorías sobre las que tratan los artículos publicados sobre enfoques de aprendizaje.

\begin{tabular}{|c|c|c|}
\hline CATEGORÍA & DESCRIPCIÓN & $\begin{array}{l}\text { ARTÍCULOS } \\
\text { PUBLICADOS }\end{array}$ \\
\hline 1 & Caracterización de los enfoques de aprendizaje & 14 \\
\hline 2 & $\begin{array}{l}\text { Distinción entre enfoques de aprendizaje y otros construc- } \\
\text { tos similares y definición conceptual }\end{array}$ & 5 \\
\hline 3 & Correlación de enfoques de aprendizaje y género & 2 \\
\hline 4 & $\begin{array}{l}\text { Correlación de enfoques de aprendizaje y contextos cultu- } \\
\text { rales }\end{array}$ & 1 \\
\hline 5 & $\begin{array}{l}\text { Correlación entre enfoques de aprendizaje y rendimiento } \\
\text { académico }\end{array}$ & 15 \\
\hline 6 & Correlación entre enfoques de aprendizaje y titulación & 2 \\
\hline 7 & $\begin{array}{l}\text { Validación de instrumentos utilizados para caracterizar } \\
\text { enfoques de aprendizaje }\end{array}$ & 4 \\
\hline 8 & Correlación entre enfoques de aprendizaje y ocio & 1 \\
\hline 9 & $\begin{array}{l}\text { Correlación de enfoques de aprendizaje y enfoques de } \\
\text { enseñanza }\end{array}$ & 5 \\
\hline 10 & Correlación entre enfoques de aprendizaje y TIC & 2 \\
\hline
\end{tabular}


CATEGORíA

11

12
DESCRIPCIÓN

ARTÍCULOS

PUBLICADOS

Correlación entre enfoques de aprendizaje con familia e institución

Correlación entre enfoques de aprendizaje y disposición para estudiar

1

2

En la tabla 9, se observa que en dos de las doce categorías se concentran la mayoría de los artículos; ellas son, de un lado, la relación entre los enfoques de aprendizaje y el rendimiento académico de una muestra de interés, con 15 artículos publicados (Abalde, Barca, Muñoz y Zeimer, 2009; Valle, González, Núñez y González, 1998; Valle., et al., 2000; Barca, Do Nascimento, Brenlla, Porto, 2008; Barca, Fernández y Mejía, 2011; Barca, Regina, Brenlla y Santamaría, 2000; De la Fuente, Pichardo, Justicia y Barbén, 2008; González, Del Rincón y Bayot, 2010; Muñoz y Gómez, 2005; Recio y Cabero, 2005, Ruiz, Hernádez y Ureña 2008; Salas, 1998; Soto, 2012; Gallardo, Suárez y Ferreras, 2007).

De otro lado, caracterizar los enfoques de aprendizaje en una muestra de interés para el investigador, con 14 artículos publicados (Cumplido, Campos, Chávez, García, 2005; Esquivel, Rodríguez y Padilla, 2009; García, De la Fuente, Justicia y Pichardo, 2005; Gargallo, Suárez, García, Pérez y Sahuquillo, 2012; Hernández, 1993; Hernández, García, Martínez, Hervás y Maquilón, 2002; Hernández, Rodríguez y Ruiz, 2010; Montealegre y Núñez, 2009; Paz et al., 2001; Salas, Santos y Parra, 2004; Salim, 2004; Salim y Lotii, 2011; Valle, González, Vieiro, Cuevas, Rodríguez, Baspino, 1997).

Posteriormente, se ubican, con cinco publicaciones, artículos en los que se establecen las relaciones entre los enfoques de aprendizaje de un grupo de estudiantes y los enfoques de enseñanza de sus docentes (González, 2010; González, Montenegro, López, Munita y Collao 2011; Olmedo, 2013; Riveros, Bernal y González 2011; Trillo y Méndez, 1999).

También son cinco los artículos publicados interesados en realizar la distinción y revisión conceptual entre los enfoques de aprendizaje con constructos similares como los estilos de aprendizaje y los estilos cognitivos (Barca y Brenilla, 2006; González, 1997; López Aguado. y López Alonso., 2013; López Aguado y Silva, 2009; Marín, 2001).

Finalmente, hay cuatro artículos en que se revisan, traducen, adaptan y/o validan los instrumentos con que se caracterizan los enfoques de aprendizaje en contextos específicos (Barca Lozano, Peralbo y Brenlla, 2004; González, Rincón, y Rincón, 2011; Hernández, García y Maquilón, 2004; Romero, Dolores, González, Carrillo, Pedraja, García y Pérez, 2013). 
Las restantes siete categorías, muestran uno o dos artículos publicados, que establecen relaciones entre los enfoques de aprendizaje y estas categorías. A continuación se examinan los artículos de las tres categorías en que mayoritariamente se han centrado estas investigaciones.

\section{Relación entre enfoques de aprendizaje y rendimiento académico}

En términos generales, en estos artículos se identifica el enfoque de aprendizaje en una muestra de estudiantes en un contexto particular, a través de alguno de los instrumentos que se han diseñado para ello; posteriormente, toman los resultados de las evaluaciones obtenidas por cada estudiante, para, mediante procedimientos estadísticos, establecer las correlaciones entre el enfoque de aprendizaje y el rendimiento académico mostrado. Los autores también buscan las causas que generan los resultados encontrados y proponen intervenciones educativas, cuando ven la necesidad.

En su mayoría, las publicaciones señalan que la adopción de un enfoque profundo de aprendizaje se relaciona de forma positiva con el rendimiento académico, y que, los estudiantes de bajo rendimiento académico tienen una concepción sobre el estudio; este hecho los lleva, mediante una motivación de naturaleza extrínseca, a enfrentar su aprendizaje con estrategias propias del enfoque superficial. Allí se plantean intervenciones tendientes a mejorar esta concepción y a replantear las estrategias de aprendizaje.

\section{Caracterización de los enfoques de aprendizaje}

Los 14 artículos que se encontraron en esta categoría, toman mayoritariamente como muestra estudiantes universitarios, y caracterizan el enfoque de aprendizaje de acuerdo con diferentes titulaciones, profesores y asignaturas. Un aspecto que se analiza en este tipo de publicaciones, es la consistencia que se presenta entre motivación y estrategias de los estudiantes que conforman la muestra, así como la influencia del contexto en la adopción de uno u otro enfoque de aprendizaje. También se comparan los resultados obtenidos con los arrojados por las investigaciones realizadas por los autores pioneros de las escuelas de Gotemburgo, Edimburgo y Sídney.

Por su parte, las conclusiones apuntan al diseño de programas de intervención que propendan por minimizar aquellos aspectos que inducen la adopción de un enfoque superficial, y en maximizar aquellos que promueven el uso de un enfoque profundo. También sugieren la planeación e implementación de programas que potencien técnicas de estudio y que favorezcan una ruta profunda en el aprendizaje. 


\section{Relación entre el enfoque de aprendizaje y el enfoque de enseñanza}

Las temáticas aquí se centran en la premisa de Biggs (2005) en relación a que en un proceso de enseñanza/aprendizaje lo que realmente importa es lo que el estudiante hace, más que lo que el docente hace. En este sentido, se busca establecer las posibles correlaciones entre el enfoque de aprendizaje de los estudiantes (superficial o profundo), y el enfoque de enseñanza de los profesores (transmisión de información centrada en el profesor o cambio conceptual centrado en el estudiante).

Los resultados tienden a presentar orientaciones al actuar de los docentes en su ejercicio profesional, que permitan un alineamiento entre el enfoque de aprendizaje de los estudiantes, el de enseñanza de los profesores y los resultados del proceso educativo. Todos los documentos analizados han estado centrados en el ámbito universitario.

\section{Validación de instrumentos utilizados para caracterizar enfoques de aprendizaje}

Estos artículos han traducido los cuestionarios originales utilizados para caracterizar enfoques de aprendizaje, en especial el ASSIST (Tait, Entwistle y McCune, 1998) de Entwistle, el R-SPQ2F (Biggs, Kember y Leung, 2001) y el R-LPQ-2F (Kember, Biggs y Leung, 2004), para adaptarlos, validarlos y aplicarlos en los diferentes contextos. Las traducciones del inglés al español, la adaptación y posterior validación, se consignan en detalle, se reportan los resultados estadísticos y se comparan con los de los autores originales. También se ponen a prueba en estudios piloto.

\section{Otras temáticas tratadas}

Las restantes categorías, en las que se muestran uno o dos artículos, establecen vínculos entre enfoques de aprendizaje con diferentes variables como género (Buendía y Olmedo, 2002; Cano, 2000), ocio (López Alonso, López-Aguado, González, y Fernández, 2012), titulación (Corominas, Tesouro y Teixidó, 2006), contextos culturales (Buendía y Olmedo, 2003), contextos familiares e institucionales (Reyes, 2003), uso de TIC (Carrascal, Alvarino y Díaz, 2009; Maquilón, Mirete, García y Hernández Pina, 2013), entre otros. 
En cuanto al género, una publicación muestra que no hay evidencia empírica para establecer una preferencia de enfoque de aprendizaje entre mujeres y hombres; otra muestra que en las mujeres, prevalece el miedo al fracaso, como sentimiento negativo que la impulsa a orientar sus procesos de aprendizaje mediante un enfoque superficial, mientras que en los hombres prevalecen las actitudes negativas ante el estudio, con el mismo efecto de elegir un enfoque superficial.

En lo relativo a los contextos culturales, un artículo muestra que estos están influenciando decididamente el proceso de enseñanza/ aprendizaje y por tanto el enfoque de aprendizaje.

Por su parte, y mediante cálculos de correlación y de regresión múltiple, en la titulación se constata un vínculo entre enfoques de aprendizaje y factores de personalidad, que predicen la elección de una carrera universitaria; por ello se hace énfasis en una adecuada y oportuna orientación del alumnado a la hora de realizar la selección de la carrera, pues esta determinará las motivaciones y estrategias que definirán el enfoque de aprendizaje.

De la misma manera, la relación entre enfoque de aprendizaje y ocio, se da en la confirmación de la hipótesis que apunta a que: los estudiantes que presentan un enfoque de aprendizaje predominantemente profundo, percibirán disponer de menos tiempo de ocio que aquellos que presentan un enfoque más superficial, debido al carácter competidor de ambas actividades.

Para las publicaciones relacionados con las TIC, los resultados obtenidos permiten establecer que una valoración positiva de su uso, se relaciona positivamente con un enfoque profundo de aprendizaje, y que las experiencias de aula mediadas por TIC, son valoradas positivamente por parte de los estudiantes.

En lo concerniente al trabajo en enfoques de aprendizaje y familia e institución, se analiza la adopción de los diferentes enfoques atribuibles a las "relaciones familia/centro", en las que se generalizan las valoraciones que la familia hace del estudio, su satisfacción con el rendimiento del hijo, los refuerzos familiares, las expectativas, etc. Esto determina el tipo de motivación intrínseca o extrínseca que lleva al estudiante a adoptar un enfoque específico.

Por último, la disposición para el estudio es un factor muy influyente en el enfoque de aprendizaje, ya que los resultados muestran relaciones significativas entre estrategias de disposición al aprendizaje, y utilización de enfoque de aprendizaje profundo, y entre estas variables y la cantidad de horas de dedicación semanal (Pérez, Valenzuela, Díaz, González y Núñez, 2011). De otro lado, esta disposición determina procesos metacognitivos, de autonomía y autorregulación que favorecen un enfoque profundo de aprendizaje (Soler y Moreno, 2012). 


\section{Discusión y conclusiones}

La línea de investigación sobre los enfoques de aprendizaje surgió en la década del setenta, desde un paradigma puramente cualitativo; luego, en la década del ochenta, y debido a la proliferación de inventarios diseñados para su caracterización, tomó también una perspectiva desde el paradigma cuantitativo. Son numerosas las investigaciones que se han venido adelantando en los últimos veinte años en idioma español. En ellas, el propósito es analizar el proceso de enseñanza/aprendizaje desde la perspectiva del estudiante más que desde la del docente. Es aquí donde la teoría de los enfoques de aprendizaje cobra gran importancia, por su naturaleza bidireccional en el sentido que, de un lado, es imperativo tener en cuenta la influencia del contexto académico y la naturaleza de los contenidos que se han de aprender en la adopción de uno u otro enfoque; de otro lado, estos enfoques pueden conformar una evidencia directa de la calidad del proceso educativo.

La revisión que se presenta en este documento sobre la teoría de los enfoques de aprendizaje permite ver la relevancia del constructo en las dos direcciones señaladas al final del párrafo anterior, pues los 54 artículos analizados y enmarcados en una de las 12 categorías, tienen todos en común, un interés por investigar: a) de qué manera los estudiantes aprenden, b) mediante qué intervenciones educativas se puede propiciar que lo hagan mejor e incrementen su rendimiento académico y c) cómo propiciar que los procesos de enseñanza, aprendizaje y evaluación se alineen, de modo que no le dejen opción a los estudiantes de orientar sus procesos por un enfoque superficial.

Esta revisión bibliométrica ha permitido la adhesión a la conclusión de Hederich (2013) cuando manifiesta que es en el ámbito universitario, en general, y en las carreras de medicina y ciencias de la salud, en particular, donde mayores aportaciones y evidencias se han encontrado, apoyando la investigación sobre los enfoques de aprendizaje, y su estrecha relación con la adquisición de conocimientos de tipo funcional, más que declarativo; y el mejoramiento en el rendimiento académico de los estudiantes.

Es interesante ver cómo el análisis hace latente el incremento de investigaciones en la línea SAL en América Latina, y que los investigadores comienzan a considerar ámbitos educativos diferentes al universitario donde centrar sus trabajos. Allí 
el constructo enfoques de aprendizaje puede hacer grandes aportes a la meta fijada por las políticas públicas, de cerrar cada vez más la brecha del analfabetismo, pero con una educación, esta vez, con una calidad basada en la evidencia.

\section{Referencias bibliográficas}

Abalde, P.E.; Barca A.; Muñoz C. J. y Ziemer, M.F. (2009). Rendimiento académico y enfoques de aprendizaje: una aproximación a la realidad de la enseñanza superior brasileña en la región norte. Revista de Investigación Educativa 27(2), 303-319.

Ariza, T. y Quevedo-Blasco, R. (2013). Análisis bibliométrico de la Revista de Investigación Educativa (2000- 2012). Revista de Investigación Educativa 31 (1), 31-52.

Barca, A.; Regina, P.C.; Brenlla B. J. y Santamaría C.S. (2000). Enfoques de aprendizaje, estilos atribucionales y rendimiento académico en una muestra de alumnos de educación secundaria de Brasil. Actas $V$ congreso Galego-Portugués. de Psicopedagoxía 6(4), 1138-1663.

Barca, A.; Regina, P.C.; Brenlla, B. J. y Santamaría C. S. (2000). Factores de atribución causal, enfoques de aprendizaje y rendimiento académico en el alumnado de educación secundaria de Galicia: datos para un aná- lisis correlacional. Actas V congreso Galego-Portugués de Psicopedagoxía 6 (4), 1138-1663.

Barca, A.; Peralbo, U. y Brenlla, B.J. (2004). Atribuciones causales y enfoques de aprendizaje: la escala SIACEPA. Psicoterma 16(1), 94-103.

Barca, A. y Brenlla, B.J. (2006). Un modelo bifactorial para la explicación de los motivos y estrategias de aprendizaje en las tareas de estudio con alumnado de educación secundaria. Revista Galego-Portuguesa de Psicoloxía e Educación 13(12), 1138-1663.

Barca, A.; Do Nascimento, M.S.; Brenlla J.; Porto, R. A. y Barca Enríquez E. (2008). Motivación y aprendizaje en el alumnado de educación secundaria y rendimiento académico: Un análisis desde la diversidad e inclusión educativa. Revista AMAzônica 1(1), 10-49.

Barca, A.; Fernández, A. y Mejía, R. (2011). Autoconcepto y enfoques de aprendizaje: sus efectos en el rendimiento académico en alumnado universitario de república dominicana. Revista Galego-Portuguesa de Psicoloxía e Educación 19(2), 1138-1663.

Biggs, J.B. (2005). Calidad del aprendizaje universitario. (Trad. P. Manzano,) Madrid: Narcea.

Biggs, J.B.; Kember, D. y Leung, D. (2001). The revised two factor study processquestionnaire: 
R-SPQ-2F. British Journal of Educational Psyhology, 71, 133149.

Buendía, E.L. y Olmedo, M.E. (2002). El género: ¿constructo mediador en los Enfoques de aprendizaje universitario? Revista de Investigación Educativa 20 (2), 511-524.

Buendía, E.L. y Olmedo, M. E. (2003). Estudio transcultural de los enfoques de aprendizaje en educación superior. Revista de Investigación Educativa 21 (2), 371-386.

Cano, G.F. (2000). Diferencias de género en estrategias y estilos de aprendizaje. Psicothema 12 (3), 360-367.

Carrascal, T.N.; Alvarino, B.G. y Díaz, B. (2009). Estrategias mediadas por tic para el desarrollo de enfoque de aprendizaje profundo en estudiantes universitarios. Folios, 29, 3-18.

Corominas, R.E.; Tesouro, M.C. y Teixidó, S.J. (2006). Vinculación de los enfoques de aprendizaje con los intereses profesionales y los rasgos de personalidad. Aportaciones a la innovación del proceso de enseñanza y aprendizaje en la educación superior. Revista de Investigación Educativa 24 (2), 443-473

Cumplido, H.G.; Campos, A.F.; Chávez L. A. y García P. V. (2006). Enfoques de aprendizaje que utilizan médicos residentes en el desarrollo de una especialidad médica. Medigraphic 44(4), 321-328.

De la Fuente, J.; Pichardo, M.; Justicia, F. y Berbén, A. (2008). Enfoques de aprendizaje, autorregulación y rendimiento en tres universidades europeas. Psicothema 20(4), 705-711.

Esquivel, C.J.; Rodríguez, N.M. y Padilla, M.V. (2009). Enfoques hacia el aprendizaje. Motivos y estrategias de estudiantes de las carreras de enfermería, ingeniería y organización deportiva. Revista de Pedagogía 30(87), 309-331.

García, B.A.; De la Fuente, A.J.; Justicia, J.F. y Pichardo, M.M. (2005). Análisis del aprendizaje del profesorado en formación: ¿pertenecen sus enfoques de aprendizaje a un continuo? Revista interuniversitaria de formación del profesorado 19(3), 255-268.

Gargallo, L.B.; Suárez, R.J. y Ferreras, R.A. (2007). Estrategias de aprendizaje y rendimiento académico en estudiantes universitarios. Revista de Investigación Educativa 25(2), 421-441.

Gargallo, L.B.; Suárez, R.J.; García F.E.; Pérez P.C. y Sahuquillo M.P. (2012). Enfoques de aprendizaje en estudiantes univer- 
sitarios excelentes y en estudiantes medios. Revista Española de Pedagogía, 252, 185-200.

González, G.J.; Rincón, I.B. y Rincón, I.D. (2011). Estructura latente y consistencia interna del R-SPQ-2F: reinterpretando los enfoques de aprendizaje en el EEES. Revista de Investigación Educativa 29(2), 277-293.

González, C. (1997). Concepciones y enfoques de aprendizaje. Revista de Psicodidáctica, 4, 5-39.

González, C. (2010). El aprendizaje y el conocimiento académico sobre la enseñanza como claves para mejorar la docencia universitaria. Calidad en la educación, 33, 123-146.

González, C.; Montenegro, H.; López, L.; Munital, I. y Collao, P. (2011). Relación entre la experiencia de aprendizaje de estudiantes universitarios y la docencia de sus profesores. Calidad en la educación 35, 21-49.

González, G. J.; Del Rincón B. y Bayot, M.A. (2010). Enfoques de aprendizaje y rendimiento académico en educación secundaria. Revista Galego-Portuguesa de Psicoloxía e Educación 18(1), 1138-1663.

Hederich, C. (2013). Estilística Educativa. Revista Colombiana de Educación, 64, 21-56.

Hernández, F. (1993). Evaluación de los enfoques de aprendizaje en alumnos de octavo de E.G.B.
Revista de orientación educativa vocacional 4(5). 99-110.

Hernández, F.; García M.P. y Maquilon S.J. (2001). Estudio empírico de los enfoques de aprendizaje de los estudiantes universitarios en función del perfil de su titulación (profundo vs. superficial). Revista Española de Orientación y Psicopedagogía 12(2), 303-318.

Hernández, F.; García S.M.; Martínez, C.M., Hervás, A.R. y Maquilón, J. (2002). Consistencia entre motivos y estrategias de aprendizaje en estudiantes universitarios. Revista de Investigación Educativa 20(2), 487-510.

Hernández, F.; García, S. M. y Maquilón S. J. (2004). Análisis del cuestionario de procesos de estudio-2 factores de Biggs en estudiantes universitarios españoles. Revista fuentes, 6, 96-114.

Hernández, F.; Rosario, P.; Cuesta, S.; Záez, J.D.; Martínez, C.P. y Ruiz, L.E. (2006). Promoción del aprendizaje estratégico y competencias de aprendizaje en estudiantes de primero de universidad: evaluación de una intervención. Revista de Investigación Educativa 24(2), 615-631.

Hernández, F.; Rodríguez, N.M. y Ruiz, L.E. (2010). Enfoques de aprendizaje en estudiantes universitarios. Revista Iberoamericana de Educación 53(7), 1-11.

Hernández, F.; Maquilón, J.; y Monroy, H.F. (2012). Estudio de los 
enfoques de enseñanza en profesorado de educación primaria. Profesorado: Revista de curriculum y formación del profesorado 16(1), 61 - 67.

Kember, D.; Biggs, J. y Leung, D. (2004). Examining the muntidimensionality or approaches to learnisn though the development of a revised version of the Learning Process Questionnaire. British Journal of Educational Psychology, 74, 261-280.

López Aguado, M. y Silva F.E. (2009). Estilos de aprendizaje. Relación con motivación y estrategias. Revista Estilos de Aprendizaje 4(4), 4-24.

López Alonso, A.; López Aguado, M.; González M. y Fernández, M.E. (2012). El ocio y los enfoques de aprendizaje en estudiantes universitarios de Enfermería. Revista de Investigación Educativa 30 (1), 53-70.

López Aguado, M. y López Alonso, A. (2013). Los enfoques de aprendizaje. Revisión conceptual y de investigación. Revista Colombiana de Educación, 64, 131-153.

Marín, M. (2002). La investigación sobre diagnóstico de los estilos de aprendizaje en la enseñanza superior. Revista de investigación educativa 20(2), 303-337.

Maquilón, J.; Mirete, A.B.; García, F.A. y Hernández, F. (2013). Valoración de las TIC por los estudiantes universitarios y su relación con los enfoques de aprendizaje. Revista de investigación educativa 31(2), 537-554.

Marton, F. y Säljö, R. (1976a). On qualitative differences in learning: 1. Outcome and process. British Journal of Educational Psychology, 46, 4-11.

Marton, F. y Säljö, R. (1976b). On quialitative differences in learning: 2. Outcome as a function of the leamer's conception of the task. British Journal of Educational Psychology, 46,115-217.

Montealegre, G. y Núñez, M. (2009). ¿Tienen aprendizaje de calidad los estudiantes de medicina? Acta médica Colombiana 34(3), 122-127.

Muñoz, E. y Gómez, G. (2005). Enfoques de aprendizaje y rendimiento académico de los estudiantes universitarios. Revista de Investigación Educativa 23(2), 417-432.

Olmedo, E.M. (2013). Enfoques de aprendizaje de los estudiantes y metodología docente: Evolución hacia el nuevo sistema de formación e interacción propuesta en el EEES. Revista de Investigación Educativa 31(2), 411-429. 
Paz, E.; Muñoz, C.M.; Colbs, L.; Buendía, E.L.; Olmedo, M.E.; Berrocal, E.; Cajide, V.J.; Soriano, A.E.; Hernández, F.; García, S.M. y Maquilón, S.J. (2001). Los enfoques de aprendizaje en estudiantes Universitarios españoles. Revista de Investigación Educativa 19(2), 465-4.

Pérez, V.A.; Valenzuela, M.; Castellanos, A.; Díaz, M.J.; González-Pienda, J. y Núñez, P.J. (2011). Disposición y enfoques de aprendizaje en estudiantes universitarios de primer año. Universitas Psychologica 10(2), 441-449.

Recio, S.M. y Cabero, A.J. (2005). Enfoques de aprendizaje, rendimiento académico y satisfacción de los alumnos en formación en entornos virtuales. Pixel-Bit Revista de medios y Educación, 25, 93-115.

Reyes, G.C. (2003). Familia, centro y enfoques de aprendizaje. Revista Galego-Portuguesa de Psicoloxía e Educación 8(7), 1138-1663.

Riveros, E.; Bernal M. y González N. (2011). Prevalencia de los enfoques de aprendizaje en estudiantes de fisiología médica: Cuestionario de procesos de estudio revisado de dos factores (R-SPQ-2F). Biosalud 10(2), 37-47.

Romero, A.; Dolores, M.; González, F.; Carrillo, E.; Pedraja, M.; García, J. y Pérez, M. (2013). Enfo- ques de aprendizaje en estudiantes universitarios: comparación de resultados con los cuestionarios ASSIST y R-SPQ-2F. Revista de investigación educativa 31(2), 375-391.

Rosário, P.; Gracio, M.; Núñez, J. y González-Pienda J. (2006). Perspectiva fenomenográfica de las concepciones del aprendizaje. Revista Galego-Portuguesa de Psicoloxía e Educación 13(11), 1138-1663.

Rosário, P.; Lourenço, A.; Paiva, O.; Rodríguez, A.; Valle. A. y TueroHerrero E. (2012). Predicción del rendimiento en matemáticas: efecto de variables personales, socioeducativas y del contexto escolar. Psicothema 24(2), 289-295.

Ruiz, L.E., Hernández, F. y Ureña, V.F. (2008). Enfoques de aprendizaje y rendimiento institucional y afectivo de los alumnos de la titulación de ciencias de la actividad física y del deporte. Revista de Investigación Educativa 26(2), 307-322.

Salas, S.R. (1998). Enfoques de aprendizaje entre estudiantes universitarios. Estudios pedagógicos, 24, 59-78.

Salas, S.R.; Santos R.M.; Parra A.S. (2004). Enfoques de aprendizaje y dominancias cerebrales entre estudiantes universitarios. Aula abierta, 84, 3-22.

Salim, R. (2004). El cuestionario CEPEA: herramienta de evaluación de enfoques de apren- 
dizaje en estudiantes universitarios. Revista Iberoamericana de Educación. Recuperado de http://www.rieoei.org/ investigacion/1060Salim.PDF

Salim, R. y Lotii, M. (2011). Evaluación de enfoques, motivaciones y estrategias de aprendizaje en estudiantes del primer año universitario de odontología. Cuadernos de Educación 9(9), 245-260.

Soler, C.M. y Moreno G. A. (2012). Experiencia de aula basada en los enfoques de aprendizaje. Estudiantes del grado décimo fomentan el desarrollo del espíritu científico en estudiantes de preescolar. Revista Científica, 16, 118-129.

Soto, C.J. (2012). Enfoques y estrategias de aprendizaje: un binomio para comprender el rendimiento en la educación secundaria. Revista de Investigación en Educación 10(2), 95-108.

Tait, H.; Entwistle, N.J. y McCune, V. (1998). A Reconceptualization of the approaches to Studying Inventory. En: C. Rust (Ed.). Improving students as learners. pp. 262-271. Oxford: Oxford Brookes University, The Oxford Center for Staff and Learning Development.

Trillo, A. y Méndez, G.F. (1999). Modelos de enseñanza de los profesores y enfoques de aprendizaje de los estudiantes: Un estudio sobre su relación en la universidad de Santiago de Compostela. Revista de Estudios e Experiencia Educativas 14(15), 131-147.

Valle, A.A.; González, C.R.; Núñez, P.J.; Valle, A.A. y González-Pineda, J. (1998). Variables Cognitivo-Motivacionales. Enfoques De Aprendizaje Y Rendimiento Académico. Psicothema 10(2), 393-412.

Valle, A.A.; González, C.R.; Núñez P.J.; Suárez R.J.; Piñeiro A.I. y Rodríguez M. S. (2000). Enfoques de aprendizaje en estudiantes universitarios. Psicothema 12(3), 368-375.

Valle, A.A.; González, C.R.; Vieiro I.P.; Cuevas G.L.; Rodríguez M.S y Baspino F. M. (1997). Características diferenciales de los enfoques de aprendizaje en estudiantes universitario. Revista de Psicodidáctica, 4, 41-58. 